\title{
Damage classification and estimation in experimental structures using time series analysis and pattern recognition
}

\author{
Oliver R. de Lautour \\ Department of Civil and Environmental Engineering \\ The University of Auckland \\ Private Bag 92019 \\ Auckland Mail Centre \\ Auckland 1142 \\ New Zealand \\ Email: Oliver.deLautour@beca.com \\ Piotr Omenzetter (corresponding author) \\ Department of Civil and Environmental Engineering \\ The University of Auckland \\ Private Bag 92019 \\ Auckland Mail Centre \\ Auckland 1142 \\ New Zealand \\ Email: p.omenzetter@auckland.ac.nz \\ Tel.: +64 93737599 ext. 88138 \\ Fax: +64 93737462
}

\begin{abstract}
Developed for studying long sequences of regularly sampled data, time series analysis methods are being increasingly investigated for the use of Structural Health Monitoring (SHM). In this research, Autoregressive (AR) models were used to fit the acceleration time histories obtained from two experimental structures: a 3-storey bookshelf structure and the ASCE Phase II Experimental SHM Benchmark Structure, in undamaged and limited number of damaged states. The coefficients of the AR models were considered to be damage sensitive features and used as input into an Artificial Neural Network (ANN). The ANN was trained to classify damage cases or estimate remaining structural stiffness. The results showed that the combination of AR models and ANNs are efficient tools for damage classification and estimation, and perform well using small number of damage sensitive features and limited sensors.
\end{abstract}

Keywords: Structural Health Monitoring, Damage detection, Damage classification, Damage estimation, Pattern recognition, Time series analysis, Autoregressive models, Artificial Neural Networks. 


\section{Introduction}

Despite considerable research efforts that Structural Health Monitoring (SHM) has received over the past decade, robust and reliable methods capable of detecting, locating and estimating damage whilst being insensitive to changes in environmental and operating conditions have yet to be agreed upon. It appears that vibration based SHM methods are amongst the most promising. While extensive literature reviews of such methods can be found elsewhere [1,2], the focus herein is on the problems and techniques relevant to this research, namely, the applications of parametric time series methods for investigating damage in engineering systems, and the use of Artificial Neural Networks (ANNs) for recognizing patterns in responses of healthy and damaged structures.

Time series techniques, originally developed for analysing long sequences of regularly sampled data, are inherently suited to SHM. However, such techniques have yet to be fully explored. Gertler [3] discusses a model based approach to fault detection and isolation in engineering systems based on the use of parity relations and time series parameter estimation techniques. The various tactics for generation of residuals that can later be statistically tested for the presence of faults are explored. Fassois and Sakellariou [4] present an overview of the principles and techniques of time series analysis and their classification into non-parametric and parametric methods for applications in SHM. Differentiation between faulty or damaged and healthy systems was achieved within the framework of statistical hypothesis testing. As the present study uses a parametric method, the following literature review focuses mainly on such approaches. There have been a number of studies in which time series models were used as auxiliary tools for identification of dynamic characteristics, usually modal parameters, later used for damage detection $[5,6]$. Several researchers examined the errors in structural responses produced by time series models fitted to data from undamaged and damaged structures and tried to detect damage [7-11]. Detection of abrupt changes, outliers and intervention analysis in time series models has also been investigated for revealing anomalies in SHM data and assessment of their impact [12-14].

Of particular relevance to the research presented herein are however investigations where the coefficients of time series models were used to form damage sensitive features. Sadeghi and Fassois [15] applied Autoregressive Moving Average models with eXogenous input (ARMAX) to dynamic signals from a laboratory beam and a numerical model of a truss and used coefficients of those models as damage sensitive features. Damage was detected and broadly localized by locating the feature vectors in the hyperspace spanned by their means and standard deviations. Feature dimensionality reduction via Principal Component Analysis (PCA) and an information entropy based criterion was also studied. Sakellariou and Fassois [16] extended the approach by observing changes in time series coefficients and used them for damage detection, localization and severity estimation in a numerical 6- 
DOF linear model of a shear type building subjected to seismic excitation. Sakellariou and Fassois [17] generalized the method using Autoregressive models with eXogenous input (ARX) whose coefficients were functions of fault magnitude and applied it to study damage in a laboratory aircraft skeleton structure. A study by Sohn et al. [18] used Autoregressive (AR) models to fit the dynamic response of a concrete bridge pier. By applying a statistical process control approach to the projected coefficients of the AR models the authors were able to distinguish between healthy and damaged states. A later study by Sohn et al. [19] applied a similar methodology to health monitoring of a surface-effect fast patrol boat. However, the authors did not attempt to locate or quantify damage. Gul et al. [20] presented a study in which AR coefficients from a laboratory steel beam with varying support conditions were classified using a clustering algorithm or a multivariate statistical technique. Omenzetter and Brownjohn [21] used a vector Seasonal Autoregressive Integrated Moving Average model to detect abrupt changes in strain data collected from the continuous monitoring of a major bridge structure. The seasonal model was used because of the strong diurnal variation in the data caused by the temperature cycle. Zheng and Mita [22-24] used Autoregressive Moving Average (ARMA) models in a two-stage damage assessment method where differences between ARMA models were calculated as their Itakura distances, model cepstra or subspace angle distances used as damage detection features, and enhanced localization performance was achieved using pre-whitening filters. The approaches were applied to numerically simulated and experimental laboratory data. Nair et al. [25] used an ARMA time series to model the vibration signals from the ASCE Phase II Experimental SHM Benchmark Structure. The authors defined a damage sensitive feature used to discriminate between the damaged and undamaged states of the structure based on the first three AR coefficients. Localization of damage was achieved by introducing another feature, also based on the AR coefficients, found to increase from a baseline value when damage was near. Nair and Kiremidjian [26] investigated Gaussian Mixture Modelling, an unsupervised pattern recognition technique, to model the first AR coefficients obtained from fitting ARMA time series to an analytical, 12-DOF model of the ASCE Phase II Experimental SHM Benchmark Structure. The extent of damage was shown to correlate well with the Mahalanobis distances between undamaged and damaged feature clusters. These two approaches were later applied to another experimental benchmark structure [27]. Finally, while the previous references were concerned with linear time series models, the use of non-linear time series for damage detection has also begun to attract attention [28-30].

For developing relationships between damage sensitive features and damage states or quantitative damage indices ANNs have widely been used. The popularity of ANNs can be attributed to their capabilities of pattern recognition, classification and function approximation. Numerous applications of ANNs for structural damage detection use spectral or modal-based damage sensitive features, in which category Ritz vectors may also be included [31-35]. Another strongly represented trend is to train ANNs to replicate time histories of structural responses and analyse the prediction errors produced by 
such networks [36-41]. A limited number of studies use parameters of ANNs themselves as damage sensitive features [42].

The contribution of this study is the development of a method that integrates the use of AR models to establish damage sensitive feature and application of ANNs for damage classification and estimation of remaining structural stiffness. The proposed method is tested on two experimental structures.

\section{Outline of damage classification and estimation method and underlying theory}

\subsection{Outline of damage classification and estimation method}

The proposed approach to damage classification and estimation can be summarized as follows:

- Structural acceleration time histories in the undamaged and various damaged states are fitted with AR models.

- The coefficients of an AR model, arranged in a vector, are chosen as damage sensitive feature.

- If required, the reduction of the dimensionality of damage sensitive feature is performed using Principal Component Analysis (PCA) or selection of subsets of AR coefficients and/or sensors.

- ANNs are trained to recognize changes in the patterns of the (possibly reduced) AR coefficients and relate them to either a specific damage state (damage classification) or remaining structural stiffness (damage estimation). The AR coefficient vectors are fed into an ANN as individual vectors even though several of them may belong to the same experiment.

The approach was applied to two experimental laboratory cases: a 3-storey bookshelf structure mounted on a shake-table, and data collected from the ASCE Phase II Experimental SHM Benchmark Structure [43]. This study was a simple case of the damage classification and estimation problem where a limited number, compared to all imaginable, damage cases and severities were introduced to both structures. Accelerations were recorded at several locations while the structures were under forced excitation. The 3-storey bookshelf structure was excited using a shake-table producing simulated earthquake motions. The ASCE Phase II Experimental SHM Benchmark Structure was excited using an electro-dynamic shaker producing white noise. In both cases, the ANNs were shown to be able to classify damage or estimate the remaining stiffness with good accuracy; for the ASCE Phase II Experimental SHM Benchmark Structure this was also demonstrated to be true while using small numbers of sensors and reduced size feature.

The following sections describe briefly the rather well known underlying theory of techniques used in this study: AR time series models, ANNs, experimental modal analysis, model updating, and PCA.

\subsection{Autoregressive models}


In this research, AR time series models are used to describe the acceleration time histories. AR models are a staple of time series analysis [44] and are often used in the analysis of stationary time series processes. A stationary process is a stochastic process, one that obeys probabilistic laws, in which the mean, variance and higher order moments are time invariant. AR models attempt to account for the correlations of the current observation in time series with its predecessors. A univariate AR model of order $p$, or $\operatorname{AR}(p)$, for the time series $\left\{x_{t}\right\}(t=1,2, \ldots, n)$ can be written as:

$$
x_{t}=\phi_{1} x_{t-1}+\phi_{2} x_{t-2}+\ldots+\phi_{p} x_{t-p}+a_{t}
$$

where $x_{t}, \ldots x_{t-p}$ are the current and previous values of the series, $\phi_{1}, \ldots \phi_{p}$ are AR coefficients, and $\left\{a_{t}\right\}$ is a Gaussian white noise error time series with a zero mean.

\subsection{Artificial Neural Networks}

To classify damage sensitive features of the signals, in this case AR coefficients, coming from the undamaged and damaged structure ANNs are used. ANNs are structures capable of pattern recognition, classification and function approximation tasks and have been used extensively in the civil engineering field [45]. ANNs utilizing the supervised error Back-Propagation (BP) training algorithm [46] are commonly referred to as BP neural networks. BP networks are the most popular ANN and have been used in this study.

The structure of a single hidden layer BP network is shown in Fig. 1, where $\mathbf{x}$ and $\mathbf{o}$ are the input and output vectors of the network, the neurons are denoted by circles and the bias inputs, denoted by squares, are taken as +1 . The weights for the whole network, denoted by vector $\mathbf{w}$, store information and are learnt during the training process.

The error $E$ in the network is a function of the weights and can be written as:

$$
E(\mathbf{w})=\frac{1}{2} \mathbf{e}(\mathbf{w})^{T} \mathbf{e}(\mathbf{w})
$$

where $\mathbf{e}(\mathbf{w})$ is an error vector defined by:

$$
\mathbf{e}(\mathbf{w})=\mathbf{d}-\mathbf{o}(\mathbf{w})
$$

d is the vector of target values or desired network outputs, and superscript $T$ denotes transposition. In training, the error in the network was minimized by iteratively adjusting weights according to the BP algorithm. The Levenberg-Marquardt algorithm [47] was used instead of the gradient descent method 
used in the original BP algorithm to update the weights. This modification to the BP algorithm is described in [48]. Introducing the Jacobian matrix $\mathbf{J}$ defined as:

$$
\mathbf{J}(\mathbf{w})=\frac{\partial \mathbf{e}}{\partial \mathbf{w}}
$$

the new weights can be found through the application of the following iterative process:

$$
\mathbf{w}_{k+1}=\mathbf{w}_{k}-\left[\mathbf{J}_{k}^{T} \mathbf{J}_{k}+\lambda_{k} \mathbf{I}\right]^{-1} \mathbf{J}_{k}^{T} \mathbf{e}_{k}
$$

where subscript $k$ denotes the iteration step. The parameter $\lambda_{k}$ is a scalar that controls convergence properties. If $\lambda_{k}$ is equal to zero, the Levenberg-Marquardt algorithm becomes the Gauss-Newton method.

\subsection{Experimental modal analysis}

Although the proposed damage identification method does not use modal parameters as damage sensitive features, experimental modal analysis was conducted for the 3 -storey bookshelf structure in order to obtain an insight into the system dynamics and to estimate modal parameters for updating of the computer models of the structure in various damage states. The natural frequencies, viscous damping ratios and mode shapes were determined from system identification of a discrete state-space model. A discrete state space model at time step $k$ can be written as follows:

$$
\begin{aligned}
& \mathbf{x}_{k+1}=\mathbf{A} \mathbf{x}_{k}+\mathbf{B} \mathbf{u}_{k} \\
& \mathbf{y}_{k}=\mathbf{C} \mathbf{x}_{k}+\mathbf{D} \mathbf{u}_{k}
\end{aligned}
$$

where $\mathbf{x}_{k}, \mathbf{u}_{k}$ and $\mathbf{y}_{k}$ are respectively the state, input and output vectors at time $k$, and $\mathbf{A}, \mathbf{B}, \mathbf{C}$ and $\mathbf{D}$ are system matrices to be determined in the identification process. In this case, the output vector was the three storey accelerations and the input vector was the shake table acceleration. The Prediction Error Method identification algorithm implemented in the system identification toolbox in MATLAB [49] was used to estimate the system matrices $\mathbf{A}, \mathbf{B}, \mathbf{C}$ and $\mathbf{D}$.

\subsection{Model updating}

In order to match experimental results, analytical modals can be updated on a range of experimental responses including natural frequencies, mode shapes and frequency domain data [50]. Natural frequencies are often the most certain modal parameters obtained from modal analysis and are often 
used in the model updating process, as in this study. The error $\mathbf{e}_{i}$ between the analytical and experimental frequencies, $\omega_{a, i}$ and $\omega_{e, i}$, can be expressed as an error vector:

$$
\mathbf{e}_{i}=\frac{\omega_{a, i}^{2}-\omega_{e, i}^{2}}{\omega_{e, i}^{2}}
$$

where subscript $i$ refers to the $i$-th mode. The analytical frequencies depend on a set of parameters, such as masses and member stiffnesses, which define the model and are denoted by vector $\theta$. In the sensitivity-based updating process [50], the vector of errors for all considered modes $\mathbf{e}$ is minimized by an iterative procedure where the updating parameters $\theta_{k}$ at iteration step $k$ are adjusted using

$$
\boldsymbol{\theta}_{k+1}=\boldsymbol{\theta}_{k}-\mathbf{S}_{k}^{+} \mathbf{e}_{k}
$$

where $\mathbf{S}^{+}$is the pseudoinverse of the sensitivity matrix whose entries can be evaluated as:

$$
\mathbf{S}_{i j}=\frac{1}{\omega_{e, i}^{2}} \boldsymbol{\phi}_{a, i}^{T}\left[\frac{\partial \mathbf{K}}{\partial \boldsymbol{\theta}_{j}}-\omega_{a, i}^{2} \frac{\partial \mathbf{M}}{\partial \boldsymbol{\theta}_{j}}\right] \boldsymbol{\phi}_{a, i}
$$

where $\mathbf{K}$ and $\mathbf{M}$ are the stiffness and mass matrices and $\phi_{a, i}$ are the analytical mode shape vectors.

\subsection{Principal Component Analysis}

PCA is a popular multivariate statistical technique often used to reduce multidimensional data sets to lower dimensions [51]. Given a set of $p$-dimensional vectors $\mathbf{x}_{i}(i=1, \ldots, n)$ drawn from a statistical distribution with mean $\overline{\mathbf{x}}$ and covariance matrix $\Sigma$, PCA seeks to project the data onto a new $p$ dimensional space with orthogonal coordinates via a linear transformation.

Decomposition of the covariance matrix by singular value decomposition leads to:

$$
\Sigma=\mathbf{V} \Lambda \mathbf{V}^{T}
$$

where $\Lambda=\operatorname{diag}\left[\sigma_{1}^{2}, \ldots, \sigma_{p}^{2}\right]$ is a diagonal matrix containing the eigenvalues of $\Sigma$ ranked in the descending order $\sigma_{1}^{2} \geq \ldots \geq \sigma_{p}^{2}$, and $\mathbf{V}$ is a matrix containing the corresponding eigenvectors or principal components. The transformation of a data point $\mathbf{x}_{i}$ into principal components is:

$$
\mathbf{z}_{i}=\mathbf{V}^{T}\left(\mathbf{x}_{i}-\overline{\mathbf{x}}\right)
$$


The new coordinates $\mathbf{z}_{i}$ are uncorrelated and have a diagonal covariance matrix $\Lambda$. Therefore, the entries of $\mathbf{z}_{i}$ are linear combinations of the entries of $\mathbf{x}_{i}$ which explain variances $\sigma_{1}^{2}, \ldots, \sigma_{p}^{2}$. To reduce the dimensionality, a selection $q<p$ of principal components can be used that retains those components that contribute most to the data variance, thus reducing the dimension of the data to $q$.

\section{Application to 3-storey bookshelf structure}

\subsection{Description of 3-storey bookshelf structure and data acquisition}

The 3-storey bookshelf structure used in this study was approximately $2.1 \mathrm{~m}$ high and constructed from equal angle aluminium column sections and stainless steel floor plates bolted together with aluminium brackets as shown in Fig. 2. The stainless steel plates were $4 \mathrm{~mm}$ thick and $650 \mathrm{~mm} \times 650 \mathrm{~mm}$ square. The column sections were $30 \mathrm{~mm} \times 30 \mathrm{~mm}$ equal angles. Two section thicknesses were used for the columns, either $4.5 \mathrm{~mm}$ or $3 \mathrm{~mm}$, for the undamaged and damaged states, respectively. Each column was made of $3 \times 0.7 \mathrm{~m}$ high segments, rather than one long angle, in order to make them easily replaceable for simulation of localized damage at different storeys. The column sections were fastened at each end with two M6 bolts to aluminium brackets (Fig. 2b). Additional brackets were installed at the base of the structure to minimize torsional motion. The whole structure was mounted on a $20 \mathrm{~mm}$ plywood sheet fixed with M10 bolts to the shake table.

The structure was instrumented with four $2.5 \mathrm{Vg}^{-1}$ uniaxial accelerometers, one for measuring the table acceleration and one for each storey. Accelerations were measured in the direction of ground motion at $400 \mathrm{~Hz}$ using a computer fitted with a data-logging card. All data were filtered with a zero phase shift $50 \mathrm{~Hz}$ low pass filter. Afterwards the data was decimated by a factor of four for modal analysis and eight for time series modelling. This reduced the original $400 \mathrm{~Hz}$ signal down to $100 \mathrm{~Hz}$ and $50 \mathrm{~Hz}$, respectively. The decimate procedure implemented in MATLAB [52] uses an eight order Chebyshev Type I lowpass filter with cutoff frequency $(0.8 / R) \times\left(F_{s} / 2\right)$, where $F_{s}$ is the initial sampling frequency and $\mathrm{R}$ is the decimate factor, before resampling the data. The decimate process improved the quality of the data by further removing high frequency noise.

\subsection{Preliminary tasks: modal identification, model updating and time series analysis}

The proposed damage classification and estimation method was first applied to the 3-storey bookshelf structure. As the first task, the modal parameters for the undamaged structure were estimated from five response records comprising four $10 \mathrm{~s}$ and one 20 s record. In all cases, the excitation or input was Gaussian white noise. Table 1 shows the averaged experimental natural frequencies $f_{e}$ and damping ratios $\xi_{e}$ obtained from the five records. The lower and upper bounds correspond to two standard deviations or $95 \%$ confidence levels. It can be seen that while natural frequencies show little error, the 
damping ratios were much more uncertain. The mode shapes were estimated from the 20 s record only. Table 2 gives the mode shapes normalized for a maximum value of 1.00 .

Damage was introduced into the structure by replacing all four columns at a particular storey (or storeys) with thinner ones. Four damage states were considered; these were labelled D0, D1, D2 and D3 corresponded to no damage (i.e. healthy structure), $1^{\text {st }}$ storey damage, $2^{\text {nd }}$ storey damage, and simultaneous $1^{\text {st }}$ and $2^{\text {nd }}$ storey damage, respectively. Modal experiments and analyses, similar as for the healthy structure (damage state D0), were conduced and Table 3 lists the experimental frequencies $f_{e}$ in all four damage states. Table 3 also gives percentage changes of the frequencies $\Delta f_{e}$ in states D1, $\mathrm{D} 2$, and $\mathrm{D} 3$ in relation to $\mathrm{D} 0$.

In this study, the lateral stiffness of the structure in both the damaged and undamaged states could not easily be determined from analytical investigations. Initial experiments showed that the stiffness was much smaller than it would appear taken into account sectional properties of the columns and Young's modulus of aluminium. It was, however, observed that the structure lost it stiffness because of the way the column-floor joints were constructed (Fig. 2b). The use of relatively flexible mounting brackets combined with the lack of continuity of the column over the joint led to a considerable lateral stiffness loss. Precise analytical calculation of the stiffness of the joint was difficult. However, the knowledge of stiffness values is very useful. Firstly, it is necessary when one wants to estimate experimental damage as a reduction in stiffness, as done in this study. Secondly, as discussed later in Conclusions in a realworld application a computer model would have to be relied upon as it is highly unlikely that a fullscale structure would be damaged to obtain AR coefficients. Although it is outside the scope of this paper, an accurate computer model of the structure would allow AR coefficients to be calculated at various simulated damage scenarios.

For the purpose of experimental stiffness estimation, simple 3-DOF lumped mass-spring analytical models were updated on the experimental natural frequencies obtained from the experimental modal analysis. The updating parameters were chosen to be the lateral stiffness of each storey $k_{1}, k_{2}$, and $k_{3}$. The initial stiffnesses were estimated by rough hand calculations giving $9300 \mathrm{~N} / \mathrm{m}$ for $k_{2}$ and $k_{3}$. Because of the additional brackets placed at the base of the structure, $k_{1}$ was expected to be significantly greater than $k_{2}$ or $k_{3}$, and a value for $k_{1}$ of $40000 \mathrm{~N} / \mathrm{m}$ was used. Additionally, after the updating process was complete, the analytical mode shapes were checked against the experimental mode shapes using Modal Assurance Criterion (MAC) [50]. Table 4 lists the results from the updated analytical models. The analytical frequencies $f_{a}$ are very close to their experimental counterparts reported previously in Table 3. The MAC values show excellent correlation for the $1^{\text {st }}$ mode, and the $2^{\text {nd }}$ and $3^{\text {rd }}$ modes show good correlation. The updated storey stiffnesses are given in Table 5. Finally, Table 6 quantifies damage at each storey for all damage states D0-D3 as the ratio of remaining to initial lateral stiffness. 
For the purpose of simulating structural response, eight scaled earthquake records were used to excite the test structure on the shake table in the four damage states. Table 7 lists the earthquakes used, the peak ground acceleration (PGA) of the original and scaled records, the duration of the record and the frequency at which the earthquake was sampled. The earthquakes were scaled so that a range of response amplitudes was obtained, while ensuring no yielding of the structure occurred. Yielding of the structure would cause nonlinearities in the response and was outside the scope of this study.

The acceleration time history of each storey was modelled using a univariate AR model. In this study, the optimal AR model was selected using the well known Akaike Information Criterion (AIC) [53]. AIC provides a trade off between the accuracy of a model in tracing the actual data and model order. Figure 3 shows an example of variation of AIC with increasing model order for acceleration from 3storey bookshelf structure. A plateau of smallest values can be seen for orders between 23 and 28 . Based on an analysis of AIC plots for different storeys and response time histories AR(24) models were adopted for each storey. The AR coefficients were estimated from a 500-point window advancing 100 points until the end of the record was reached. Additional observations of the tracking of AR coefficients for different window advancing steps showed that the selected step was adequate for capturing the system dynamics. Although the structural response due to earthquake motion would normally be considered as nonstationary, analysis of small segments only can satisfactorily preserve stationarity [54]. Model diagnostic checking, comprising testing the stationarity and normality of the residuals and significance of sample residual autocorrelation function and partial autocorrelation function, was conducted according to the procedures outlined in [44]. The Kolmogorov-Smirnov test [55] was used to test for normality of the residuals and the Ljung-Box statistic [44] was used to test for correlation. The tests indicated that the residuals conformed to a Gaussian white noise process. Figure 4 shows an example of the comparison between the actual and one-step ahead prediction of accelerations for 3-storey bookshelf structure using an AR(24) model, confirming a very good accuracy of the adopted AR model order.

For the purpose of damage classification and estimation studies, a data set of 388 points (vectors of AR coefficients) containing 97 points for each damage state was obtained. The data set was then randomly divided into 300 points for training and 88 points for testing the ANNs, ensuring approximately equal numbers of damage features from each damage state (for the testing data these numbers were 21 for state D0, 23 for state D1, 21 for state D2, and 23 for state D3, respectively). During the training and testing of the ANNs damage feature vectors were fed into the ANNs as individual vectors even though several of them belonged to the same experiment.

\subsection{Damage classification}


Initially, an ANN was trained to distinguish between the four damage states only, i.e. to perform a simple damage classification task. The damage states D0, D1, D2 and D3 were assigned the target vector outputs $\left[\begin{array}{llll}1 & 0 & 0 & 0\end{array}\right]^{T},\left[\begin{array}{llll}0 & 1 & 0 & 0\end{array}\right]^{T},\left[\begin{array}{llll}0 & 0 & 1 & 0\end{array}\right]^{T}$ and $\left[\begin{array}{llll}0 & 0 & 0 & 1\end{array}\right]^{T}$, respectively. The input vectors comprised all the AR coefficients from three storeys. Several single hidden layer ANNs with different numbers of neurons in the hidden layer and initial weights values were tried. A single hidden layer ANN with four hidden layer neurons was adopted. This ANN was found to give good results with only three or 3.4\% of misclassifications when applied to all 88 testing data points. Two and one misclassified features came from states D2 and D3, respectively.

\subsection{Estimation of remaining stiffness}

Rather than simply classifying damage into several classes, a more useful approach would give information about the remaining structural stiffness. In this section, the ANNs were trained to relate the AR coefficients to the ratio of remaining to initial lateral stiffness at each storey, listed in Table 6. A single hidden layer ANN with five hidden layer neurons was found to give good predictions. The results have been shown graphically in Figure 5, where the estimated remaining stiffness for the testing data has been plotted separately for all four damage states and all three storeys with the actual stiffness indicated with a horizontal line. For perfect predictions all the data points should lie on these lines. The means of the identified ratios together with two standard deviation bounds are shown in Table 8 and show good agreement with the actual stiffness obtained by modal updating. At a $95 \%$ confidence level the estimated values do not differ from the actual values by more than $3.2 \%$. Best estimation results were obtained from damage state D0 with an error of $1.5 \%$ averaged over all storeys. The damage state D2 proved to have the most error with an average of $1.9 \%$. These results show that the ANN has correctly estimated the damage at each story with only a small amount of scatter about the ratio of remaining to initial lateral stiffness.

\section{Application to ASCE Phase II Experimental SHM Benchmark Structure}

\subsection{Description of the structure and experimental programme}

The ASCE Phase II Experimental SHM Benchmark Structure [43] is a 4-storey, 2-bay by 2-bay steel frame with a $2.5 \mathrm{~m} \times 2.5 \mathrm{~m}$ floor plan and a height of $3.6 \mathrm{~m}$ (Fig. $6 \mathrm{a}$ ). Note only a brief description of the structure, test setup and test programme is given herein; a full description can be found at the benchmark problem website. The columns were $\mathrm{B} 100 \times 9$ sections and the floor beams were $\mathrm{S} 75 \times 11$ sections, all sections were Grade 300 steel. The beams and columns were bolted together. Bracing was added in all bays with two $12.7 \mathrm{~mm}$ diameter threaded steel rods, see Fig. 6b. Additional mass was distributed around the structure to make it more realistic. Four 1000kg floor slabs were placed on the 
$1^{\text {st }}, 2^{\text {nd }}$ and $3^{\text {rd }}$ floors, one per bay. On the $4^{\text {th }}$ floor, four $750 \mathrm{~kg}$ slabs were used. Two of the slabs per floor were placed off-centre to increase the coupling between translational and torsional motion.

In the following discussions the locations in the structure are referred to using their respective geographical directions of north $(\mathrm{N})$, south $(\mathrm{S})$, east (E) and west (W).

A total of nine damage scenarios were simulated on the structure, these involved the removal of bracing and the loosening of bolts in the floor beam connections. Table 9 lists the damage states and gives a description of damage. The different configurations give a mixture of minor and extensive damage cases.

A series of ambient and forced vibration tests were carried out on the structure. Of primary interest in this study were the forced random vibration tests conducted using an electro-dynamic shaker mounted on the SW bay of the $4^{\text {th }}$ floor on the diagonal. Input into the shaker was band-limited $5-50 \mathrm{~Hz}$ white noise. The structure was instrumented with 15 accelerometers; three accelerometers each for the base, $1^{\text {st }}, 2^{\text {nd }}, 3^{\text {rd }}$ and $4^{\text {th }}$ floors. These were located on the $\mathrm{E}$ and $\mathrm{W}$ frames to measure motion in the N-S direction and in the centre to measure the E-W motion. Acceleration data was recorded at $200 \mathrm{~Hz}$ using a data acquisition system and filtered with anti-aliasing filters.

\subsection{Damage classification using reduced size feature}

In this study, the data from the ASCE Phase II Experimental SHM Benchmark Structure served a twofold purpose. Firstly, they were used to validate the performance of the proposed method on a more realistic and complex structure with complex damage scenarios. Secondly, the problem of data reduction for multi-sensor SHM system proved to be of importance and was addressed.

Because quantitative information about the damage severity was not readily available, in this application damage classification into the nine states was attempted. The nine damage configurations were assigned vector outputs from $\left[\begin{array}{lllllllll}1 & 0 & 0 & 0 & 0 & 0 & 0 & 0 & 0\end{array}\right]^{T}$ to $\left[\begin{array}{lllllllll}0 & 0 & 0 & 0 & 0 & 0 & 0 & 0 & 1\end{array}\right]^{T}$ for configurations 1 to 9 , respectively. The optimal model order was selected using AIC and the residual error series was checked for confirmation of a Gaussian white noise process following the same process as described earlier. Univariate AR(30) models were adopted and fitted to the acceleration data from each of the 15 accelerometers. The AR coefficients were estimated using least squares from 1000-point segments advancing 200 points until the end of the record was reached. A data set of 1035 feature vectors was obtained, 115 from each configuration and randomly divided into 700 for training and 335 for testing, ensuring approximately equal numbers of damage features from each damage state (for the testing data these numbers were $39,36,35,39,40,36,37,32$ and 31 for damage configurations 1 through 9 , respectively). 
Using AR(30) models and all 15 accelerometers gave the ANN input dimension of 450. A BP ANN with a 450-dimensional input proved to be difficult to train due to computational limitations. Two data reduction techniques were investigated: (i) selection of subsets of AR coefficients and/or accelerometers, and (ii) projection of the data onto a lower dimensional space using PCA.

Reducing the number of AR coefficients and/or accelerometers may adversely affect information contained in the data and degrade the performance of damage classification. Therefore several combinations of reduced AR coefficients and accelerometers were systematically investigated to ascertain their practical minimum numbers. The number of AR coefficients was reduced by selecting the first few coefficients only. This was chosen to be either (i) the first coefficient, (ii) the first two coefficients, (iii) the first three coefficients, (iv) the first four coefficients, or (v) the first six coefficients. Similarly, the number of accelerometers and their locations was either (i) the full set (15 accelerometers), (ii) omitted accelerometers located on the base and those of the $\mathrm{W}$ face measuring the $\mathrm{N}-\mathrm{S}$ motion (eight accelerometers), or (iii) same as case (ii) but with all remaining accelerometers on storeys 1 and 3 omitted (four accelerometers). A single hidden layer ANN with five hidden layer neurons was selected for its good performance. The results were analyzed as a classification task and are shown in Figure 7 where the number of misclassifications out of the 335-point test data set is given for different combinations of reduced AR coefficients and/or accelerometers. Establishing the value of a threshold where the performance of a SHM system can be judged as good or otherwise is always rather arbitrary. Here, $5 \%$ or less of misclassifications was considered to be a good result and $1 \%$ or less to be excellent. Figure 7 shows a clear boundary where performance rapidly deteriorates below the $5 \%$ misclassification threshold. The full set of 15 accelerometers achieved good performance with only one AR coefficient, while two or three AR coefficients were required for eight or four accelerometers, respectively. For excellent results at least two, three or four AR coefficients were necessary for 15, eight and four accelerometers, respectively. An overall conclusion that can be drawn from this simulation is that small numbers of AR coefficients and sensors sufficed for precise damage classification.

While the dimensionality reduction approach discussed above gave good results, a more methodical approach would retain data of statistical significance only. PCA was used to project the data onto the first 30, 20, 10, or 5 principal components. Using a single hidden layer ANN with five hidden layer neurons, the number of misclassifications with percentage errors for each damage case, when applied to testing data, is given in Table 10. The results showed good damage classification could be achieved by projection of the data onto the first 10 or more principal components. Excellent classification results were obtained when using more than 20 principal components. Some improvements over selecting subsets of AR coefficient were observed especially when the feature dimension was small. Referring to Figure 7, using four accelerometers and three AR coefficients, equating to a feature dimension of 12 , 
10 misclassifications were recorded. When using 10 principal components, five misclassifications were recorded. This comparison shows a superior performance of the more systematic feature reduction approach using PCA over simple, but subjective, selection of accelerometers and AR coefficients.

\section{Conclusions}

The methods of time series analysis were developed for understanding long, regularly sampled sequences of data and can offer new promising insights and opportunities for the SHM and damage detection research community. In this study, a method for damage classification and estimation of remaining stiffness using AR models and ANNs has been developed and applied to a simple 3-storey bookshelf structure and more complex ASCE Phase II Experimental SHM Benchmark Structure. Acceleration time histories of the structures in a small number of different, experimentally simulated damage cases and under dynamic excitation were recorded and fitted using AR models. In order to assign AR coefficients to the different damage states or estimate remaining structural stiffness BP ANNs were used.

The studies on the 3-storey bookshelf structure demonstrated that for stiffness reduction of $7 \%$ to $10 \%$ the ANN was able to classify damage correctly for $96.6 \%$ of the considered validation sets of AR coefficients. The subsequent study aiming at estimating the remaining structural stiffness of the damaged systems showed that the ANN was capable of estimating the stiffness with small errors not exceeding $3.2 \%$ of the actual values at the $95 \%$ confidence levels.

Due to computation limitations, ANNs were unable to be trained in some instances without reduction in dimensionality of the data. Two dimensionality reduction approaches were applied to the ASCE Phase II Experimental SHM Benchmark Structure to reduce the dimension of the AR coefficient feature vectors. Either a reduced numbers of AR coefficients and/or accelerometers were selected or the data was reduced using PCA. The results showed that significant reductions in dimensionality could be achieved whilst maintaining good performance of damage classification. Overall data reduction via PCA provided a small performance gain over the selection of subsets AR coefficients and/or accelerometers. Using only 10 principal components only $1.5 \%$ of misclassifications were achieved.

The proposed method faces a number of challenges that will be the focus of future studies. The method requires data from damaged states to be available. This may present a practical difficulty for the method to be applied to real life structures. However, in a real-world application data from damaged states could be obtained from an analytical model of the structure. Also, the method uses a predefined number of damage states and if presented with a feature from another damage state it will classify it incorrectly as belonging to one of those states. To overcome this problem, an ANN can be trained to estimate probabilities of the feature belonging to a given state rather than performing 'sharp' 
classification. Samples with probabilities close to one will be assigned to the respective states present in the training set while those with smaller values will not. For the rejected samples their damage states could be inferred by interpolating the ANN mapping between the damage states and the positions of feature clusters. Likewise, an ANN could be trained to interpolate the remaining structural stiffness based on the relationship learnt during training.

\section{Acknowledgements}

The authors would like to express their gratitude for the Earthquake Commission Research Foundation of New Zealand for their financial support of this research (Project No. UNI/535). Mr. Bastien Vaurigaud helped with experimental work and this contribution is also gratefully acknowledged.

\section{References}

[1] S. W. Doebling, C. R. Farrar, M. B. Prime, D. W. Shevitz, Damage identification and health monitoring of structural and mechanical systems from changes in their vibration characteristics: A literature review, LA-13070-MS, Los Alamos National Laboratory, Los Alamos, NM, USA, 1996.

[2] H. Sohn, C. R. Farrar, F. M. Hemez, D. D. Shunk, D. W. Stinemates, B. R. Nadler, A review of structural health monitoring literature: 1996-2001, LA-13976-MS, Los Alamos National Laboratory, Los Alamos, NM, USA, 2003.

[3] J. J. Gertler, Fault detection and diagnosis in engineering systems, Marcel Dekker, New York, NY, 1998.

[4] S. D. Fassois, J. S. Sakellariou, Time-series methods for fault detection and identification in vibrating structures, Philosophical Transactions of the Royal Society A: Mathematical, Physical and Engineering Sciences, 365 (2007) 411-448.

[5] C.-H. Loh, H.-M. Lin, Application of off-line and on-line identification techniques to building seismic response data, Earthquake Engineering and Structural Dynamics, 25 (1996) 269-290.

[6] J. B. Bodeux, J. C. Golinval, Application of ARMAV models to the identification and damage detection of mechanical and civil engineering structures, Smart Materials and Structures, 10 (2001) 479-489.

[7] M. L. Fugate, H. Sohn, C. R. Farrar, Vibration-based damage detection using statistical process control, Mechanical Systems and Signal Processing, 15 (2001) 707-721.

[8] H. Sohn, C. R. Farrar, Damage diagnosis using time series analysis of vibration signals, Smart Materials and Structures, 10 (2001) 446-451.

[9] Y. Lu, F. Gao, A novel time-domain auto-regressive model for structural damage diagnosis, Journal of Sound and Vibration, 283 (2005) 1031-1049.

[10] P. E. Carden, J. M. W. Brownjohn, ARMA modelled time-series classification for structural health monitoring of civil infrastructure, Mechanical Systems and Signal Processing, 22 (2008) 295-314.

[11] Q. W. Zhang, Statistical damage identification for bridges using ambient vibration data, Computers and Structures, 85 (2007) 476-485.

[12] P. Moyo, J. M. W. Brownjohn, Application of Box-Jenkins models for assessing the effect of unusual events recorded by structural health monitoring systems, Structural Health Monitoring, 1 (2002) 149-160. 
[13] P. Omenzetter, J. M. W. Brownjohn, P. Moyo, Identification of unusual events in multichannel bridge monitoring data, Mechanical Systems and Signal Processing, 18 (2004) 409430.

[14] M. Basseville, I. V. Nikiforov, Detection of abrupt changes: Theory and application, PTR Prentice-Hall, Upper Saddle River, NJ, 1993.

[15] M. H. Sadeghi, S. D. Fassois, Reduced-dimensionality geometric approach to fault identification in stochastic structural systems, AIAA Journal, 36 (1998) 2250-2256.

[16] J. S. Sakellariou, S. D. Fassois, Stochastic output error vibration-based damage detection and assessment in structures under earthquake excitation, Journal of Sound and Vibration, 297 (2006) 1048-1067.

[17] J. S. Sakellariou, S. D. Fassois, Vibration based fault detection and identification in an aircraft skeleton structure via a stochastic functional model based method, Mechanical Systems and Signal Processing, 22 (2008) 557-573.

[18] H. Sohn, J. A. Czarnecki, C. R. Farrar, Structural health monitoring using statistical process control, Journal of Structural Engineering, 126 (2000) 1356-1363.

[19] H. Sohn, C. R. Farrar, N. F. Hunter, K. Worden, Applying the LANL statistical pattern recognition paradigm for structural health monitoring to data from a surface-effect fast patrol boat, LA-13761-MS, Los Alamos National Laboratory, Los Alamos, 2001.

[20] M. Gul, F. N. Catbas, M. Georgiopoulos, Application of pattern recognition techniques to identify structural change in a laboratory specimen, in: Sensors and Smart Structures Technologies for Civil, Mechanical and Aerospace Systems 2007, San Diego, CA, 2007, pp. 65291N1-65291N10.

[21] P. Omenzetter, J. M. W. Brownjohn, Application of time series analysis for bridge monitoring, Smart Materials and Structures, 15 (2006) 129-138.

[22] H. Zheng, A. Mita, Two-stage damage diagnosis based on the distance between ARMA models and pre-whitening filters, Smart Materials and Structures, 16 (2007) 1829-1836.

[23] H. Zheng, A. Mita, Damage indicator defined as the distance between ARMA models for structural health monitoring, Structural Control and Health Monitoring, 15 (2008) 992-1005.

[24] H. Zheng, A. Mita, Localized damage detection of structures subject to multiple ambient excitations using two distance measures for autoregressive models, Structural Health Monitoring, 8 (2009) 207-222.

[25] K. K. Nair, A. S. Kiremidjian, K. H. Law, Time series-based damage detection and localization algorithm with application to the ASCE benchmark structure, Journal of Sound and Vibration, 291 (2006) 349-368.

[26] K. K. Nair, A. S. Kiremidjian, Time series based structural damage detection algorithm using Gaussian mixtures modeling, Journal of Dynamic Systems, Measurement and Control, Transactions of the ASME, 129 (2007) 285-293.

[27] H. Y. Noh, K. K. Nair, A. S. Kiremidjian, C. H. Loh, Application of time series based damage detection algorithms to the benchmark experiment at the National Center for Research on Earthquake Engineering (NCREE) in Taipei, Taiwan, Smart Structures and Systems, 5 (2009) 95-117.

[28] F. Casciati, S. Casciati, Structural health monitoring by Lyapunov exponents of non-linear time series, Structural Control and Health Monitoring, 13 (2006) 132-146.

[29] J. M. Nichols, L. N. Virgin, M. D. Todd, J. D. Nichols, On the use of attractor dimension as a feature in structural health monitoring, Mechanical Systems and Signal Processing, 17 (2003) 1305-1320.

[30] J. M. Nichols, M. Seaver, S. T. Trickey, A method for detecting damage-induced nonlinearities in structures using information theory, Journal of Sound and Vibration, 297 (2006) 1-16. 
[31] X. Wu, J. Ghaboussi, J. H. Garrett, Use of neural networks in detection of structural damage, Computers and Structures, 42 (1992) 649-659.

[32] M. F. Elkordy, K. C. Chang, G. C. Lee, Neural networks trained by analytically simulated damage states, Journal of Computing in Civil Engineering, 7 (1993) 130-145.

[33] C. C. Chang, T. Y. P. Chang, Y. G. Xu, M. L. Wang, Structural damage detection using an iterative neural network, Journal of Intelligent Material Systems and Structures, 11 (2000) 32-42.

[34] Y. Q. Ni, X. T. Zhou, J. M. Ko, Experimental investigation of seismic damage identification using PCA-compressed frequency response functions and neural networks, Journal of Sound and Vibration, 290 (2006) 242-263.

[35] H.-F. Lam, K.-V. Yuen, J. L. Beck, Structural health monitoring via measured Ritz vectors utilizing artificial neural networks, Computer-Aided Civil and Infrastructure Engineering, 21 (2006) 232-241.

[36] S. F. Masri, A. W. Smyth, A. G. Chassiakos, T. K. Caughey, N. F. Hunter, Application of neural networks for detection of changes in nonlinear systems, Journal of Engineering Mechanics, 126 (2000) 666-676.

[37] X. Jiang, H. Adeli, Pseudospectra, MUSIC, and dynamic wavelet neural network for damage detection of highrise buildings, International Journal for Numerical Methods in Engineering, 71 (2007) 606-629.

[38] X. Wu, B. Xu, K. Yokoyama, Decentralized parametric damage detection based on neural networks, Computer-Aided Civil and Infrastructure Engineering, 17 (2002) 175-184.

[39] M. Nakamura, S. F. Masri, A. G. Chassiakos, A. K. Caughey, A method for non-parametric damage detection through the use of neural networks, Earthquake Engineering and Structural Dynamics, 27 (1998) 997-1010.

[40] C. S. Huang, S. L. Hung, C. M. Wen, T. T. Tu, A neural network approach for structural identification and diagnosis of a building from seismic response data, Earthquake Engineering and Structural Dynamics, 32 (2003) 187-206.

[41] S.-L. Hung, C. Y. Kao, Detection of structural damage via free vibration responses generated by approximating artificial neural networks, Computers and Structures, 81 (2003) 2631-2644.

[42] S. Hung, C. Y. Kao, Structural damage detection using the optimal weights of the approximation artificial neural networks, Earthquake Engineering and Structural Dynamics, 31 (2002) 217-234.

ASCE Structural Health Monitoring Committee, http://cive.seas.wustl.edu/wusceel/asce.shm/.

[44] W. W. S. Wei, Time series analysis: Univariate and multivariate methods, 2nd ed, Pearson, Boston, 2006.

[45] H. Adeli, Neural networks in civil engineering: 1989-2000, Computer-Aided Civil and Infrastructure Engineering, 16 (2001) 126-142.

[46] D. E. Rumelhart, G. E. Hinton, R. J. Williams, Learning internal representations by error propagation, in: Parallel distributed processing: Explorations in the microstructures of cognition, D.E. Rumelhart and J.L. McClelland, Editors. 1986, MIT Press Cambridge, Massachusetts. p. 318-362.

[47] D. Marquardt, An algorithm for least squares estimation of nonlinear parameters, Journal of the Society for Industrial and Applied Mathematics, 11 (1963) 431-441.

[48] M. T. Hagan, M. Menhaj, Training feedforward networks with the Marquardt algorithm, IEEE Transactions on Neural Networks, 5 (1994) 989-993.

[49] L. Ljung, System identification toolbox: User's guide. Version 6, Mathworks, Natick, MA, 2006.

[50] M. I. Friswell, J. E. Mottershead, Finite element model updating in structural dynamics, Kluwer Academic Publishers, Dordrecht, 1995. 
[51] S. Sharma, Applied multivariate techniques, Wiley, New York, 1997.

[52] Mathworks, Signal processing toolbox 6: User's guide, Mathworks, Natick, MA, USA, 2008.

[53] H. Akaike, New look at the statistical model identification, IEEE Transactions on Automatic Control, AC-19 (1974) 716-723.

[54] T. Takanami, High precision estimation of seismic wave arrival times, in: The practice of time series analysis, H. Akaike and G. Kitagawa, Editors. 1999, Springer New York. p. 7994.

[55] D. Sheskin, Handbook of parametric and nonparametric statistical procedures, Chapman and Hall, Boca Raton, FL, 2003. 


\section{Table 1}

Identified frequencies and damping ratios for 3-storey bookshelf structure in healthy state.

\begin{tabular}{ccc}
\hline Mode & $f_{e}(\mathrm{~Hz})$ & $\xi_{e}(\%)$ \\
\hline $1^{\text {st }}$ & $1.93 \pm 0.01$ & $0.6 \pm 0.2$ \\
$2^{\text {nd }}$ & $5.52 \pm 0.02$ & $0.6 \pm 0.2$ \\
$3^{\text {rd }}$ & $8.55 \pm 0.04$ & $0.8 \pm 0.4$ \\
\hline
\end{tabular}

de Lautour and Omenzetter 


\section{Table 2}

Normalized mode shapes for 3-storey bookshelf structure in healthy state.

\begin{tabular}{cccc}
\hline Storey & Mode 1 & Mode 2 & Mode 3 \\
\hline $1^{\text {st }}$ & 0.18 & 0.64 & 1.00 \\
$2^{\text {nd }}$ & 0.69 & 1.00 & -0.48 \\
$3^{\text {rd }}$ & 1.00 & -0.88 & 0.16 \\
\hline
\end{tabular}


Table 3

Identified frequencies and their percentage changes for 3-storey bookshelf structure in different damage states.

\begin{tabular}{cccccccc}
\hline & \multicolumn{3}{c}{$f_{e}(\mathrm{~Hz})$} & \multicolumn{3}{c}{$\Delta f_{e}(\%)^{\mathrm{a}}$} \\
\hline Mode & $\mathrm{D} 0$ & $\mathrm{D} 1$ & $\mathrm{D} 2$ & $\mathrm{D} 3$ & $\mathrm{D} 1$ & $\mathrm{D} 2$ & $\mathrm{D} 3$ \\
\hline $1^{\text {st }}$ & 1.93 & 1.88 & 1.84 & 1.84 & $-2.5 \%$ & $-4.7 \%$ & $-4.6 \%$ \\
$2^{\text {nd }}$ & 5.52 & 5.43 & 5.46 & 5.42 & $-1.6 \%$ & $-1.0 \%$ & $-1.8 \%$ \\
$3^{\text {rd }}$ & 8.55 & 8.30 & 8.09 & 8.15 & $-2.9 \%$ & $-5.3 \%$ & $-4.6 \%$ \\
\hline
\end{tabular}

${ }^{\mathrm{a}}$ Based on D0. 


\section{Table 4}

Analytical frequencies and MAC values after updating for 3-storey bookshelf structure.

\begin{tabular}{ccccccccc}
\hline & \multicolumn{2}{c}{ D0 } & \multicolumn{2}{c}{ D1 } & \multicolumn{2}{c}{ D2 } & \multicolumn{2}{c}{ D3 } \\
\hline Mode & $f_{a}(\mathrm{~Hz})$ & MAC & $f_{a}(\mathrm{~Hz})$ & MAC & $f_{a}(\mathrm{~Hz})$ & MAC & $f_{a}(\mathrm{~Hz})$ & MAC \\
\hline $1^{\text {st }}$ & 1.92 & 1.00 & 1.91 & 0.99 & 1.85 & 1.00 & 1.84 & 0.99 \\
$2^{\text {nd }}$ & 5.49 & 0.93 & 5.47 & 0.93 & 5.46 & 0.91 & 5.45 & 0.96 \\
$3^{\text {rd }}$ & 8.54 & 0.93 & 8.28 & 0.93 & 8.46 & 0.90 & 8.20 & 0.91 \\
\hline
\end{tabular}




\section{Table 5}

Updated stiffnesses for 3-storey bookshelf structure.

\begin{tabular}{cccccc}
\hline Stiffness $(\mathrm{N} / \mathrm{m})$ & Initial estimate & D0 & D1 & D2 & D3 \\
\hline $1^{\text {st }}$ storey $k_{1}$ & $4.00 \times 10^{4}$ & $3.77 \times 10^{4}$ & $3.49 \times 10^{4}$ & $3.77 \times 10^{4}$ & $3.49 \times 10^{4}$ \\
$2^{\text {nd }}$ storey $k_{2}$ & $0.93 \times 10^{4}$ & $0.60 \times 10^{4}$ & $0.60 \times 10^{4}$ & $0.54 \times 10^{4}$ & $0.54 \times 10^{4}$ \\
$3^{\text {rd }}$ storey $k_{3}$ & $0.93 \times 10^{4}$ & $0.77 \times 10^{4}$ & $0.77 \times 10^{4}$ & $0.77 \times 10^{4}$ & $0.77 \times 10^{4}$ \\
\hline
\end{tabular}

de Lautour and Omenzetter 


\section{Table 6}

Damage at each storey as a ratio of initial stiffness for 3-storey bookshelf structure.

\begin{tabular}{ccccc}
\hline Storey & D0 & D1 & D2 & D3 \\
\hline $1^{\text {st }}$ & 1.00 & 0.93 & 1.00 & 0.93 \\
$2^{\text {nd }}$ & 1.00 & 1.00 & 0.90 & 0.90 \\
$3^{\text {rd }}$ & 1.00 & 1.00 & 1.00 & 1.00 \\
\hline
\end{tabular}




\section{Table 7}

Earthquake records used to excite 3-storey bookshelf structure.

\begin{tabular}{ccccc}
\hline Earthquake & PGA $(\mathrm{g})$ & Scaled PGA $(\mathrm{g})$ & Duration $(\mathrm{sec})$ & Sampling frequency $(\mathrm{Hz})$ \\
\hline Duzce 12/11/1999 & 0.535 & 0.027 & 25.885 & 200 \\
Erzincan 13/3/1992 & 0.496 & 0.033 & 20.780 & 200 \\
Gazli 17/5/1976 & 0.718 & 0.048 & 16.265 & 200 \\
Helena 31/10/1935 & 0.173 & 0.035 & 40.000 & 100 \\
Imperial Valley 19/5/1940 & 0.313 & 0.031 & 40.000 & 100 \\
Kobe 17/1/1995 & 0.345 & 0.035 & 40.960 & 100 \\
Loma Prieta 18/10/1989 & 0.472 & 0.047 & 39.945 & 200 \\
Northridge 17/1/1994 & 0.568 & 0.038 & 40.000 & 50 \\
\hline
\end{tabular}




\section{Table 8}

Damage estimated by ANN as a ratio of initial stiffness in 3-storey bookshelf structure.

\begin{tabular}{ccccc}
\hline Storey & D0 & D1 & D2 & D3 \\
\hline $1^{\text {st }}$ & $0.996 \pm 0.023$ & $0.927 \pm 0.027$ & $1.001 \pm 0.026$ & $0.935 \pm 0.022$ \\
$2^{\text {nd }}$ & $0.995 \pm 0.023$ & $0.997 \pm 0.021$ & $0.903 \pm 0.029$ & $0.903 \pm 0.026$ \\
$3^{\text {rd }}$ & $1.000 \pm 0.000$ & $1.000 \pm 0.000$ & $1.000 \pm 0.000$ & $1.000 \pm 0.000$ \\
\hline
\end{tabular}

de Lautour and Omenzetter 
Table 9

Damage configurations for ASCE Phase II Experimental SHM Benchmark Structure.

\begin{tabular}{cl}
\hline Configuration & Damage \\
\hline 1 & No damage \\
2 & All bracing removed on the E face \\
3 & Bracing removed on floors 1-4 on a bay on the SE corner \\
4 & Bracing removed on floors 1 and 4 on a bay on the SE corner \\
5 & Bracing removed on floors 1 on a bay on the SE corner \\
6 & All bracing removed on E face and floor 2 on N face \\
7 & All bracing removed \\
8 & Configuration 7 + loosened bolts on floors 1-4 on E face N bay \\
9 & Configuration 7 + loosened bolts on floors 1 and 2 on E face N bay \\
\hline
\end{tabular}


Table 10

Number and percentage of misclassifications using PCA reduced data for ASCE Phase II Experimental SHM Benchmark Structure.

\begin{tabular}{cc}
\hline Number of principal components & Number (and percentage) of misclassifications \\
\hline 30 & $0(0 \%)$ \\
20 & $0(0 \%)$ \\
10 & $5(1.5 \%)$ \\
5 & $70(20.9 \%)$ \\
\hline
\end{tabular}

de Lautour and Omenzetter 
Hidden layer neurons

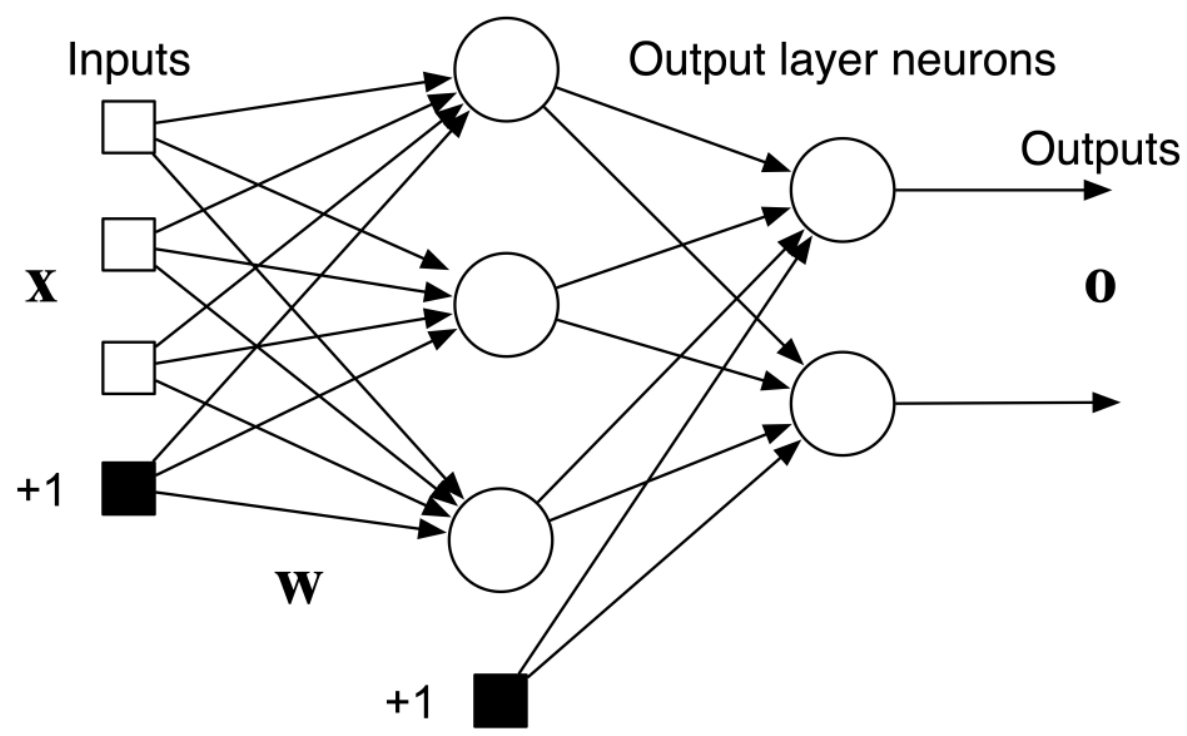

Figure 1. A single hidden layer ANN.

de Lautour and Omenzetter 


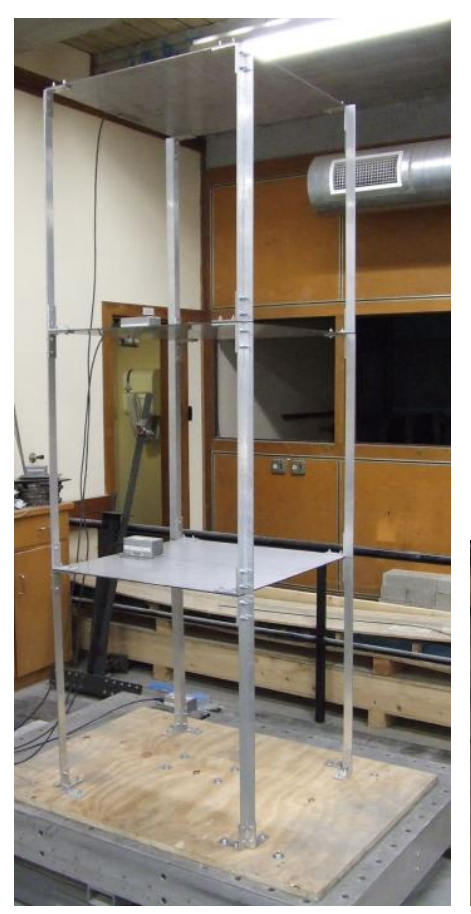

(a)

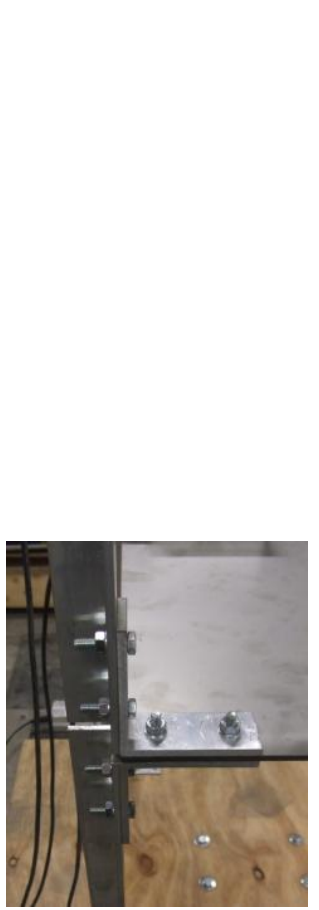

(b)

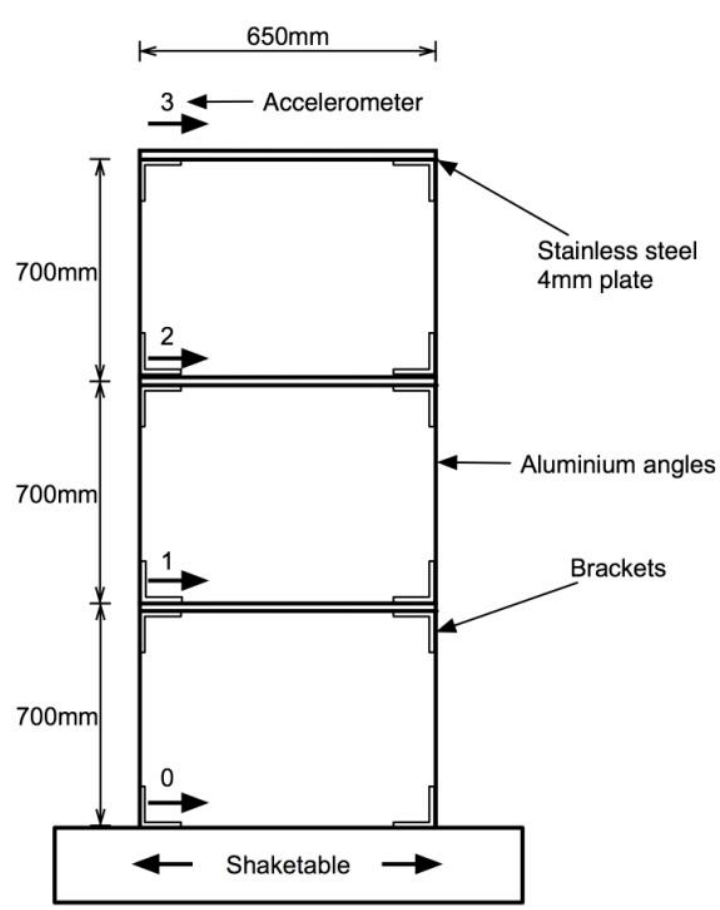

(c)

Figure 2. 3-storey bookshelf structure: (a) general view, (b) detail of column-plate joint, and (c) dimensions and accelerometer locations. 
Figure 3

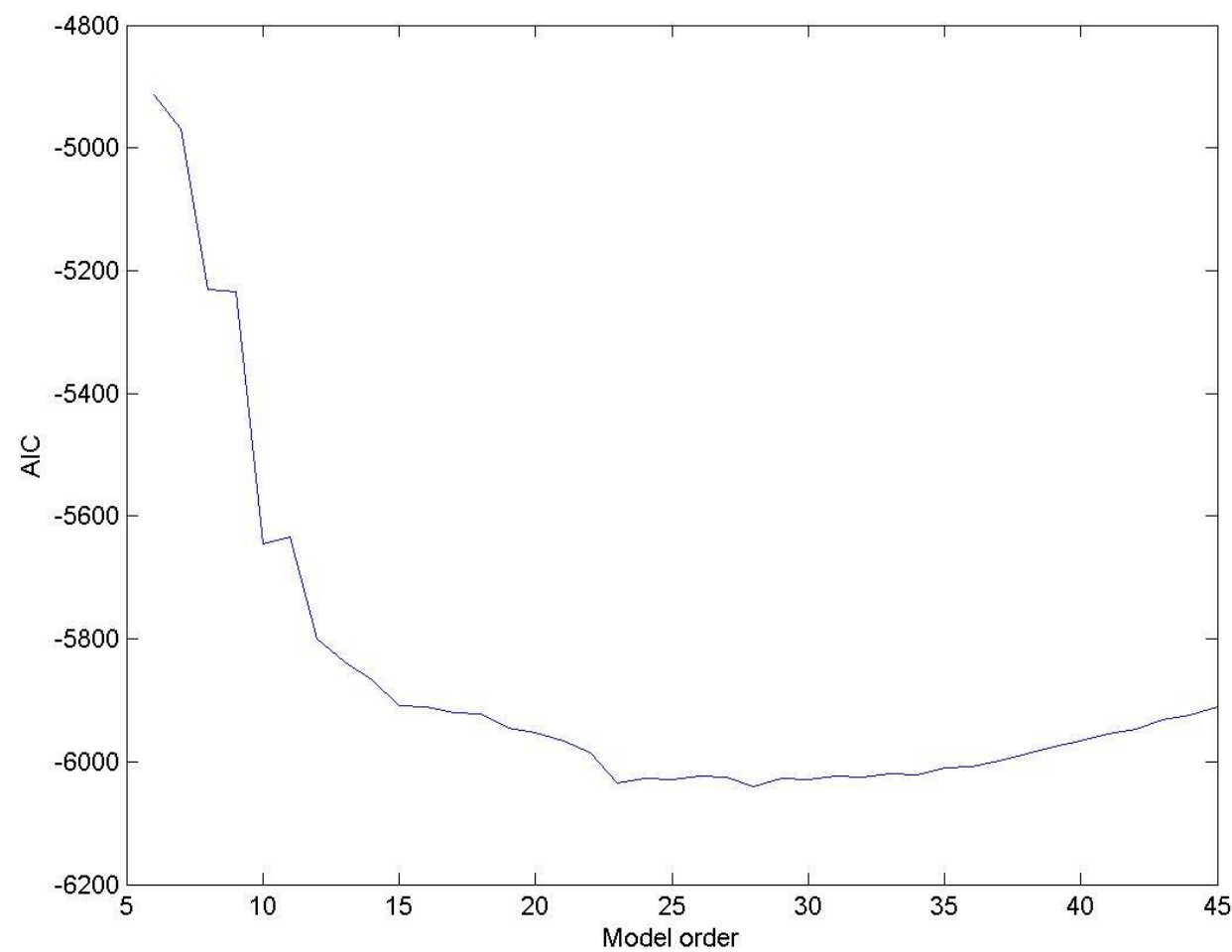

Figure 3. Variation of AIC with AR model order for 3-storey bookshelf structure. 
(a)

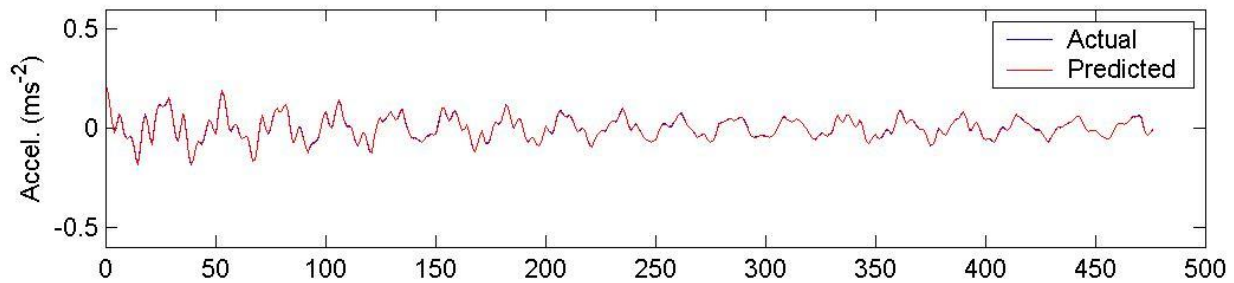

(b)

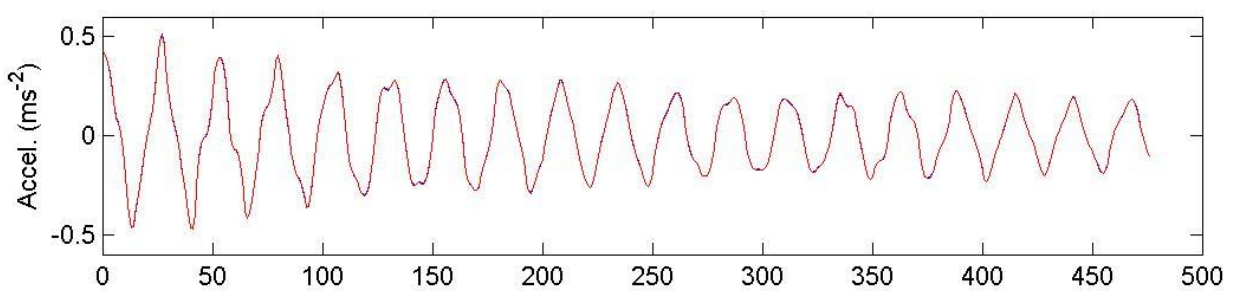

(c)

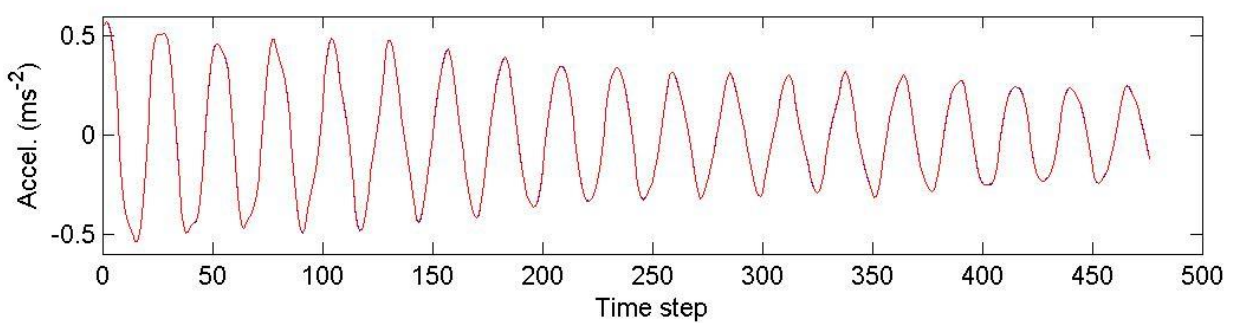

Figure 4. Actual and one-step ahead prediction of accelerations for 3-storey bookshelf structure using AR(24) model: (a) $1^{\text {st }}$ storey, (b) $2^{\text {nd }}$ storey, and (c) $3^{\text {rd }}$ storey. 
(a)

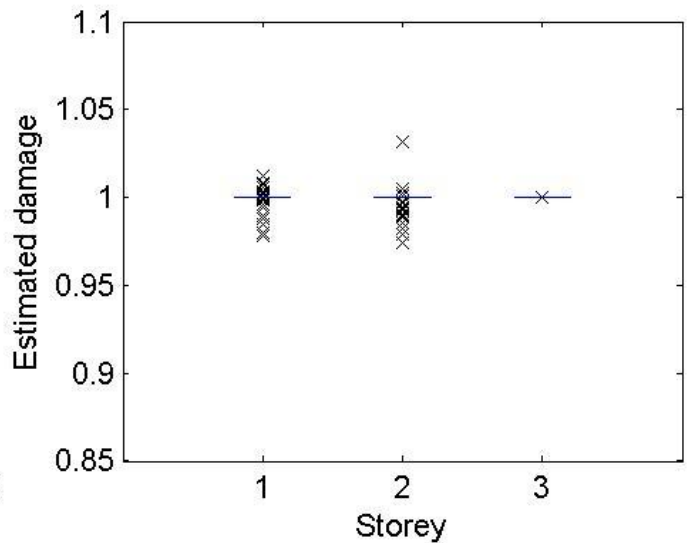

(c)

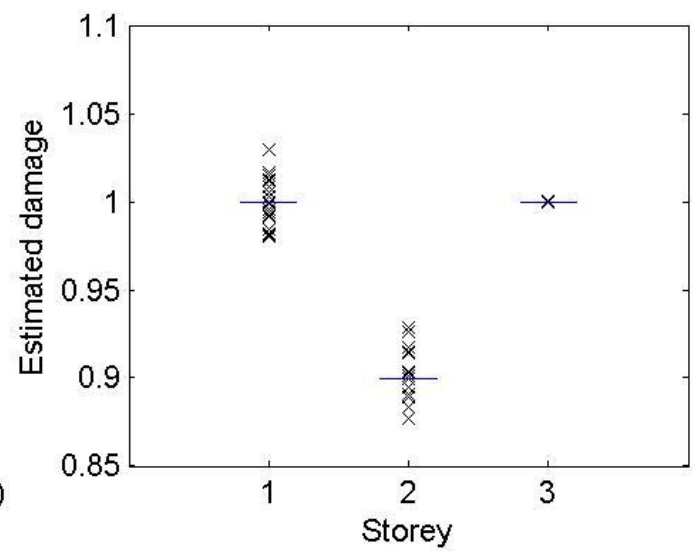

(b)

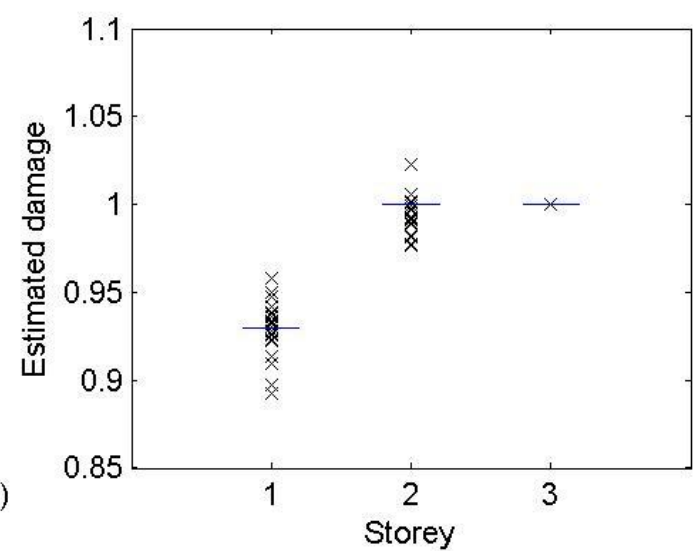

(d)

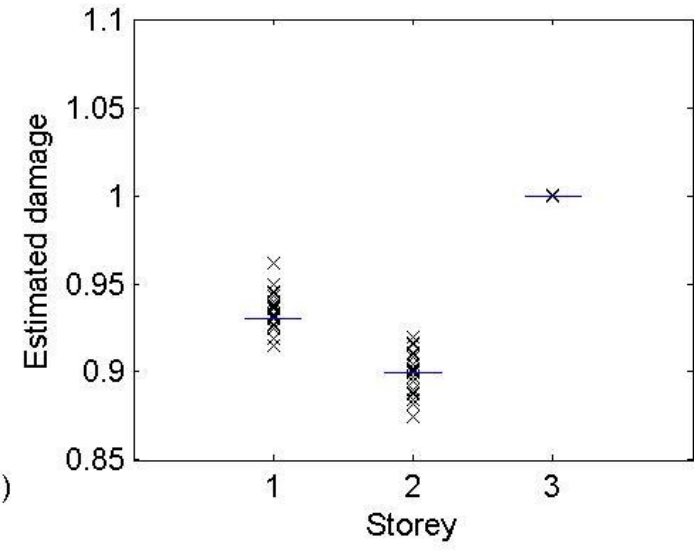

Figure 5. Estimated remaining stiffness for 3-storey bookshelf structure in states: (a) D0, (b) D1, (c) D2, and (d) D3. 


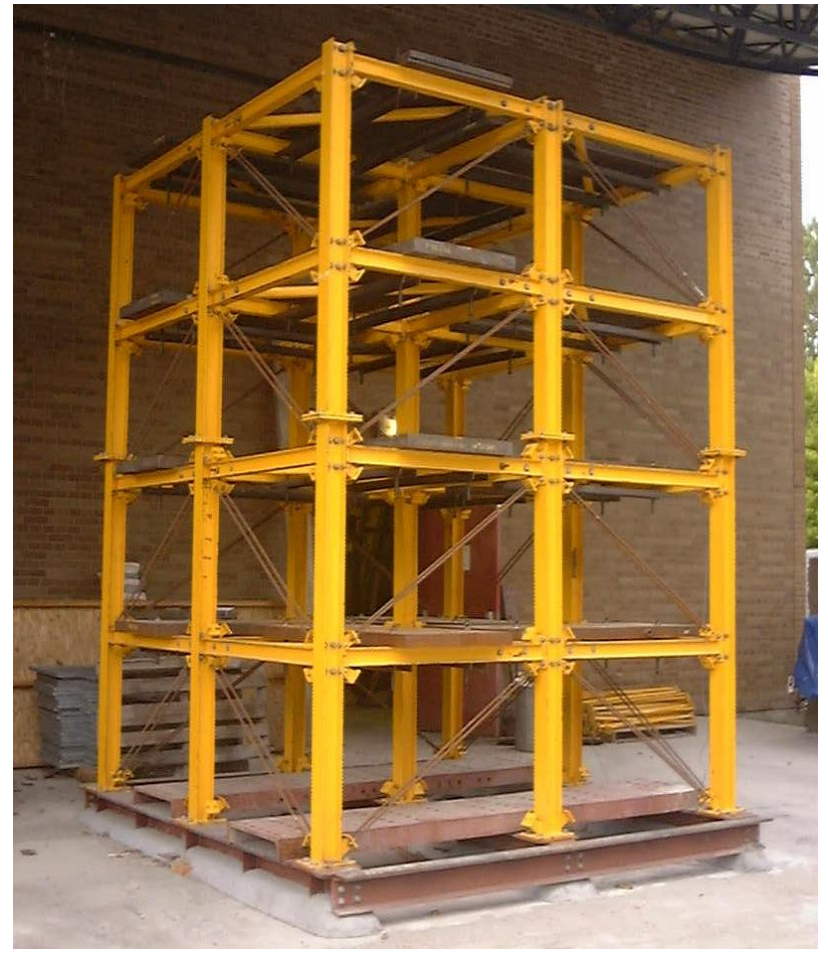

(a)

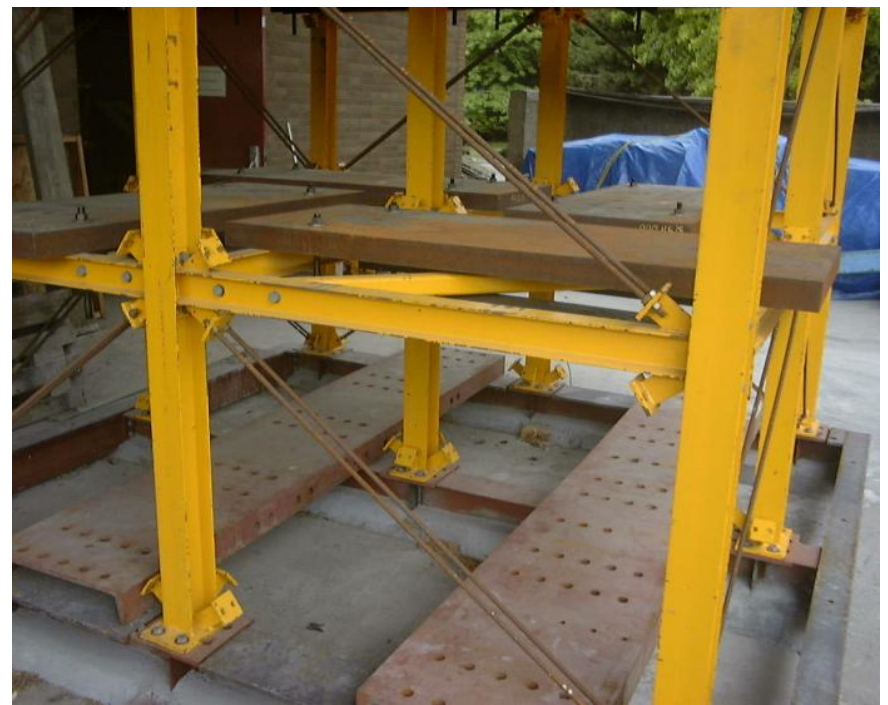

(b)

Figure 6. ASCE Phase II Experimental SHM Benchmark Structure: (a) general view, and (b) beam-column joint and bracing. 


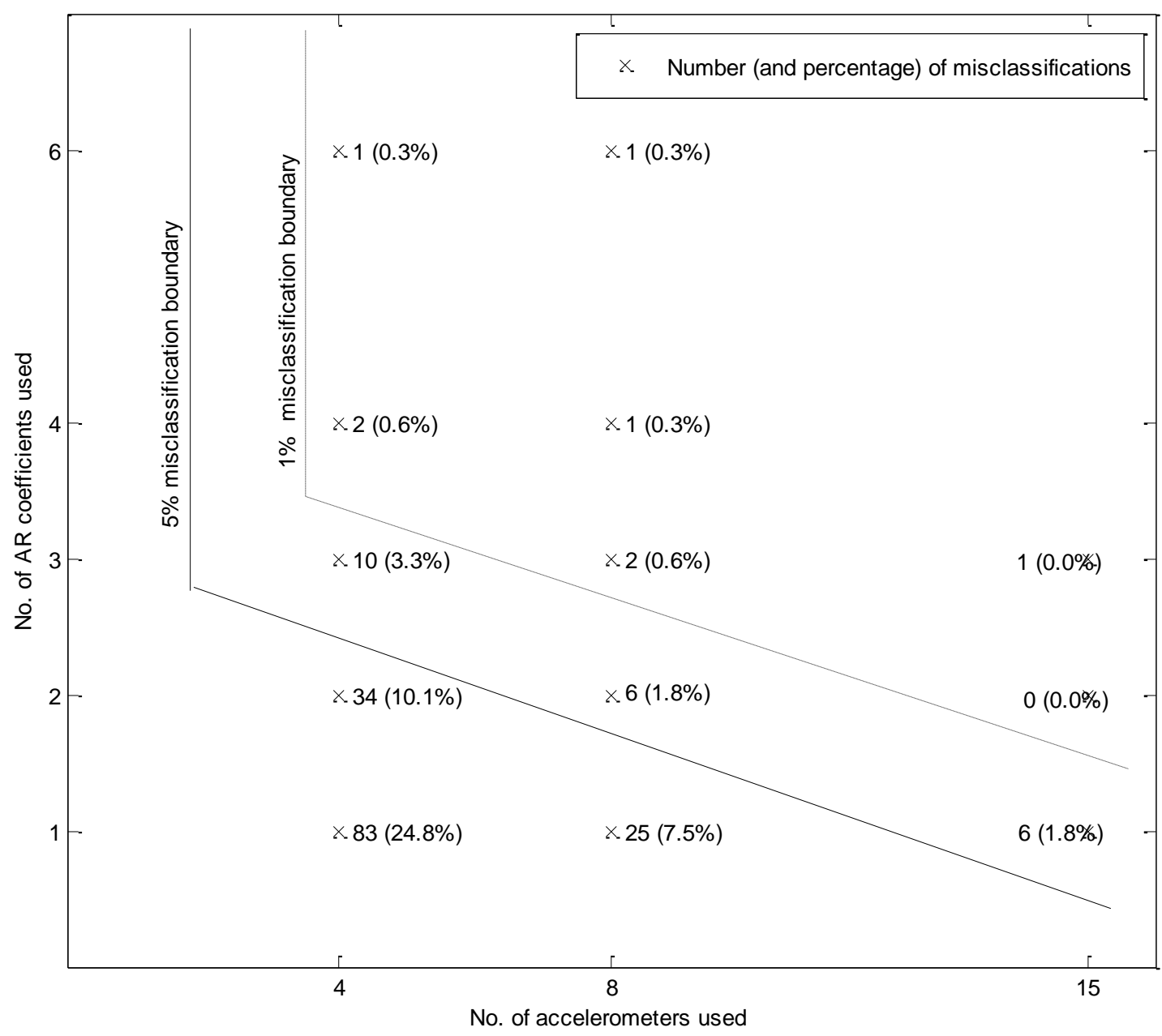

Figure 7. Number and percentage of misclassifications for the ASCE Phase II Experimental SHM Benchmark Structure using selection of subsets of AR coefficients and accelerometers. 